\title{
Editorial zum Sonderheft „Religion und Vorurteil“
}

\author{
Alexander Yendell • Yvonne Jaeckel
}

Online publiziert: 14. Mai 2020

(C) Der/die Autor(en) 2020

Neben der Frage nach der Entwicklung und Funktion von Religion in Gesellschaften hat sich in den letzten 15 Jahren in verschiedenen Fachdisziplinen die Konflikthaftigkeit von Religionen vor dem Hintergrund zunehmender religiöse Pluralität als ein gesellschaftspolitisch relevantes Thema mit Religionsbezug etabliert (z. B. Wuthnow 2005; Stolz 2005; Küpper und Zick 2010; Pollack et al. 2014; Pickel und Yendell 2016; Arweck 2016; Yendell 2018; Wäckerlig 2019) Das Thema gewinnt gegenwärtig an zusätzlicher Brisanz, da rechtspopulistische Bewegungen und Parteien teilweise erfolgreich in der Mobilisierung von Wähler*innen und Unterstützer*innen sind und die religiöse Etikettierung und Abwertung, insbesondere von Zuwanderern aus islamisch geprägten Ländern, für die Identitäts- und Migrationspolitik rechtsextremer und rechtspopulistischer Parteien bedeutend ist (Hidalgo et al. 2019).

Gleichzeitig wird vor dem Hintergrund von Pluralisierung und Globalisierung aktuell ein Wiederaufleben des Antisemitismus diskutiert (z. B. Beyer 2019; Decker et al. 2018) und die Frage gestellt, ob gerade Mitglieder unterschiedlicher religiöser Gemeinschaften unter einen verstärkten Diskriminierungsdruck geraten. Aber nicht nur die Abwertung anderer Religionsgemeinschaften, sondern auch rassistische, homophobe und sexistische Einstellungen und Verhaltensweisen sowie damit einhergehende Diskriminierungen von Minderheiten werden im religiösen Kontext thematisiert. Ein wichtiger Grund für die Thematisierung ist unter anderem, dass bestimmten Religionsgemeinschaften die Diskriminierung von Minderheiten unterstellt wird oder teilweise religiöse Lehren zur Legitimation von Diskriminierung und sozialer Abwertung missbraucht werden. In diesem Kontext kommen auch Prozesse

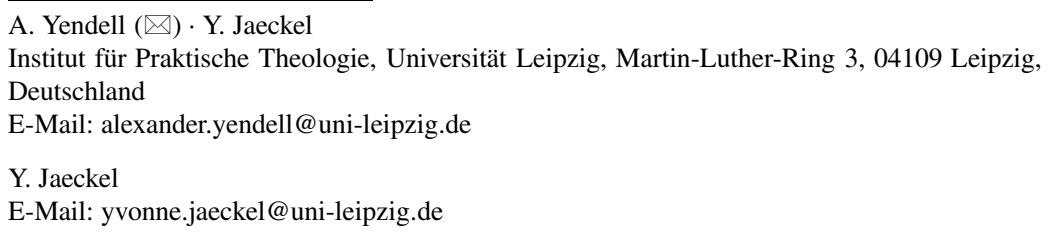


fundamentalistischer Radikalisierung wie Co-Radikalisierung in das Blickfeld der empirischen Forschung.

Neben religiösen Gemeinschaften tritt auch der Atheismus bzw. Konfessionslosigkeit gelegentlich als Objekt von Vorurteilen in den Vordergrund, fühlen sich doch immerhin 36\% der Westdeutschen und 16\% der Ostdeutschen vom Atheismus bedroht (Pollack und Müller 2013, S. 37).

Gerade an belastbarer und differenzierter empirischer Forschung mangelt es, da viele der angesprochenen Diskurse hochgradig emotional belastet und die bisherigen empirischen Erkenntnisse noch lückenhaft sind. Gelegentlich fehlt bei durchgeführten Deskriptionen der Phänomene die theoretische und konzeptionelle Tiefenschärfe. Dabei stehen gleich mehrere theoretische Zugänge zur Verfügung, die zur Erklärung der Ursachen von (gruppenbezogenen) Vorurteilen genutzt werden können. Sie können entlang einer analytischen Trennung von Mikro- und Makroebene diskutiert werden. Nicht nur gesellschaftliche Kontextfaktoren, wie verschärfte ökonomische Bedingungen vor dem Hintergrund von Globalisierung und zunehmender Wettbewerbsorientierung, sondern auch sozialstrukturelle und (sozial)-psychologische Faktoren wie Bedrohungsgefühle, autoritäre Einstellungsmuster und Identitätsbildungen kommen dabei in den Blick. Relativ wenig bekannt sind differenzierte Zusammenhänge von religionsbezogenen Variablen zu Vorurteilen. Zwar gibt es hier eine längere Wissenschaftstradition, allerdings sind die Ergebnisse noch übersichtlich und wurden zumeist nicht in größerem Zusammenhang diskutiert. Ein Grund ist, dass Studien zu Vorurteilen religionsbezogene Indikatoren häufig vernachlässigen und die verschiedenen Formen und Dimensionen individueller Religiosität nicht ausreichend berücksichtigen (Huber und Yendell 2019; Yendell und Huber 2020). Umgekehrt integrieren Studien mit einer Vielzahl von Variablen zur Religiosität häufig unzureichend die verschiedenen Theorieangebote aus dem breiten Spektrum der Vorurteilsforschung. Diesen Befund haben wir zum Anlass genommen mit empirischen Analysen stärker verschiedene Beziehungen zwischen Religion, Religiosität und Vorurteilen in den Blick zu nehmen.

\section{Hintergrund und Inhalt der Special Section}

Die in der Special Section enthaltenen Beiträge entstammen einer Tagung des Arbeitskreises quantitative Religionsforschung (AqR) zum Thema „Religion und Vorurteil“, die im Dezember 2019 an der Universität Leipzig durchgeführt wurde.

Die Beiträge geben verschiedenartige Einblicke in die zuvor aufgerissenen $\mathrm{Zu}$ sammenhänge von Religion und Vorurteilen und nutzen dabei ganz unterschiedliches Datenmaterial.

Der erste Beitrag von Kazim Celik, Gert Pickel, Julia Schuler und Oliver Decker fokussiert sich auf den Einfluss von Bedrohungswahrnehmungen auf gruppenbezogene Vorurteile und nutzt dafür die Daten des Berlinmonitor 2019. In der Studie wird der Einfluss von Ängsten und Bedrohungswahrnehmungen in Zusammenhangsanalysen auf das Vorhandensein von gruppenbezogenen Ressentiments untersucht. Interessant ist dabei der spezielle Blick auf Berlin als Hauptstadt Deutschlands und plurale Stadtgesellschaft. 
Carolin Hillenbrand untersucht Antriebs- und Immunisierungsfaktoren fremdenfeindlicher Einstellungen mit Daten des European Social Survey (ESS) für Deutschland. Im Vordergrund stehen die Annahmen, dass gemäß der sogenannten Kontakthypothese, Kontakte eine positive Wirkung hinsichtlich der Verminderung fremdenfeindlicher Einstellungen besitzen. Zugleich geht sie der Frage nach, ob Religiosität einen Fremdenfeindlichkeit befördernden Effekt aufweist.

Das Untersuchungsland Schweiz gerät bei Alexander Yendell und Stefan Huber in den Blick. Auf Datenbasis des Schweizer Haushaltspanels untersuchen sie in ihrem Beitrag den Zusammenhang von Religiosität und negativen Einstellungen gegenüber dem Islam innerhalb der Schweizer Bevölkerung. Hier zeigt sich, dass die unterschiedlichen Dimensionen von Religiosität zu unterschiedlichen Einflüssen bezüglich negativer Einstellungen gegenüber dem Islam führen.

Ein weiterer Artikel von Frank Asbrock und Monika Albrecht widmet sich speziell dem Einfluss autoritärer Einstellungen in Bezug auf die Wahrnehmung der Verletzung kultureller Normen. Dem Beitrag liegt ein experimentelles Design zugrunde und fokussiert unter anderem die Fragen, welche Faktoren autoritäre Einstellungen begünstigen und welche Rolle das Element sozialer Konformität spielt.

Heiko Beyer und Ulf Liebe beschäftigen sich in ihrer Analyse mit Gewalterfahrungen und Bedrohungswahrnehmungen von jüdischen Personen in Deutschland. Datengrundlage bildet die Antisemitismus-Erhebung der European Union Agency for Fundamental Rights, die mit zwei Erhebungszeitpunkten in den Jahren 2008 und 2018 auch Aussagen zur Entwicklung von Viktimisierungserfahrungen unter jüdischen Personen zulässt.

Ein letzter Artikel von Gert Pickel, Antonius Liedhegener, Yvonne Jaeckel, Anastas Odermatt und Alexander Yendell beschäftigt sich mit religiösen Identitäten in Deutschland und der Schweiz und deren Bezug zu gruppenbezogenen Vorurteilen. Der Beitrag nimmt dabei verschiedene Religionszugehörigkeiten, aber auch unterschiedliche Frömmigkeitsstile in den Blick.

Abschließend lässt sich resümieren, dass es sich im Bereich der Vorurteilsforschung bereits gut auf vorhandene Konzepte und Theorien zurückgreifen lässt. Allerdings scheint weiterer Forschungsbedarf hinsichtlich der Differenzierung von Ressentiments zu bestehen. So zeigte sich in den Diskussionen der Beiträge beispielsweise die Schwierigkeit, Rassismus von genereller Religionsfeindlichkeit oder spezifischer Islamophobie unterscheiden zu können. Ein anderer wichtiger Befund war der Einfluss autoritärer Einstellungen auf Vorurteile. Auch dabei wäre es zukünftig sinnvoll, weiter zu erforschen, inwiefern autoritäre Einstellungen in der Sozialisation angelegt werden oder ob sie in Kombination mit bestimmten Wertvorstellungen einhergehen.

Im Hinblick auf die Religiosität lässt sich im Einklang mit Allport festhalten, dass sie eine ambivalente Rolle einnimmt (vgl. Allport 1971). Einige Beiträge stellen nachvollziehbar dar, wie wichtig es scheint, Religiosität möglichst differenziert $\mathrm{zu}$ erfassen und in die Analyse einfließen zu lassen. So zeigen vor allem fundamentale und dogmatische Haltungen einen bestärkenden Einfluss auf Vorurteilshaltungen, personale und öffentliche Religiosität eher einen vorurteilsaubbauenden Effekt und die reine Religionszugehörigkeit besitzt oftmals keinen nennenswerten Einfluss auf Vorurteile. Auch an dieser Stelle wird es lohnenswert sein, weitere 
Analysen noch differenzierter und tiefgründiger anzugehen. Schlussendlich lässt sich auch für die Makroebene noch Forschungsbedarf anzeigen. Eine Analyse der Kontextfaktoren verschiedener Gesellschaften scheint insofern lohnenswert zu sein, als dass beispielsweise der Grad religiöser Heterogenität oder der Säkularisierungsund Segregationsgrad bislang eher selten im Zusammenhang mit Vorurteilshaltungen untersucht wird.

Wir danken allen Autorinnen und Autoren für die anregenden Diskussionen während der Tagung sowie für die Mühen, die Beiträge rechtzeitig fertigzustellen. Ebenso danken wir der Redaktion und den HerausgeberInnen der ZRGP für die reibungslose Organisation und natürlich ebenso den Gutachterinnen und Gutachtern, für die hilfreichen Kommentare.

Funding Open Access funding provided by Projekt DEAL.

Open Access Dieser Artikel wird unter der Creative Commons Namensnennung 4.0 International Lizenz veröffentlicht, welche die Nutzung, Vervielfältigung, Bearbeitung, Verbreitung und Wiedergabe in jeglichem Medium und Format erlaubt, sofern Sie den/die ursprünglichen Autor(en) und die Quelle ordnungsgemäß nennen, einen Link zur Creative Commons Lizenz beifügen und angeben, ob Änderungen vorgenommen wurden.

Die in diesem Artikel enthaltenen Bilder und sonstiges Drittmaterial unterliegen ebenfalls der genannten Creative Commons Lizenz, sofern sich aus der Abbildungslegende nichts anderes ergibt. Sofern das betreffende Material nicht unter der genannten Creative Commons Lizenz steht und die betreffende Handlung nicht nach gesetzlichen Vorschriften erlaubt ist, ist für die oben aufgeführten Weiterverwendungen des Materials die Einwilligung des jeweiligen Rechteinhabers einzuholen.

Weitere Details zur Lizenz entnehmen Sie bitte der Lizenzinformation auf http://creativecommons.org/ licenses/by/4.0/deed.de.

\section{Literatur}

Allport, Gordon W. 1971. Die Natur des Vorurteils. Köln: Kiepenheuer \& Witsch.

Arweck, Elisabeth. 2016. Young people's attitudes to religious diversity. London: Routledge.

Beyer, Heiko. 2019. The globalization of resentment: antisemitism in an inter- and transnational context. Social Science Quarterly https://doi.org/10.1111/ssqu.12649.

Decker, Oliver, Johannes Kiess, und Elmar Brähler. 2018. Antisemitische Ressentiments in Deutschland: Verbreitung und Ursachen. In Flucht ins Autoritäre: Rechtsextreme Dynamiken in der Mitte der Gesellschaft, Hrsg. Oliver Decker, Elmar Brähler, 179-216. Gießen: Psychosozial-Verlag.

Hidalgo, Oliver, Philipp W. Hildmann, und Alexander Yendell (Hrsg.). 2019. Religion und Rechtspopulismus

Huber, Stefan, und Alexander Yendell. 2019. Does Religiosity matter? Explaining right-wing extremist attitudes and the vote for the Alternative for Germany (AfD). Religion and Society in Central and Eastern Europe 15(2):63-85. https://doi.org/10.20413/rascee.2019.12.1.63-82.

Küpper, Beate, und Andreas Zick. 2010. Religion and prejudice in Europe: new empirical findings. London: Alliance Publishing Trust.

Pickel, Gert, und Alexander Yendell. 2016. Islam als Bedrohung? Beschreibung und Erklärung von Einstellungen zum Islam im Ländervergleich. Zeitschrift für Vergleichende Politikwissenschaft 10(3/4):273-309. https://doi.org/10.1007/s12286-016-0309-6.

Pollack, Detlef, Olaf Müller, Gergely Rosta, Nils Friedrichs, und Alexander Yendell. 2014. Grenzen der Toleranz: Wahrnehmung und Akzeptanz religiöser Vielfalt in Europa. Wiesbaden: Springer VS.

Pollack, Detlef, und Olaf Müller. 2013. Religionsmonitor - verstehen was verbindet. Religiosität und Zusammenhalt in Deutschland. Gütersloh. https://www.bertelsmann-stiftung.de/fileadmin/files/BSt/ Publikationen/GrauePublikationen/GP_Religionsmonitor_verstehen_was_verbindet_Religioesitaet_ und_Zusammenhalt_in_Deutschland.pdf. Zugegriffen: 30. Apr. 2020. 
Stolz, Jörg. 2005. Explaining Islamophobia. A test of four theories based on the case of a Swiss city. Swiss Journal of Sociology 31(3):547-566.

Wäckerlig, Oliver. 2019. Vernetzte Islamfeindlichkeit: Die transatlantische Bewegung gegen »Islamisierung : Events - Organisationen - Medien. Bielefeld: transcript.

Wuthnow, Robert. 2005. America and the challenges of religious diversity. Princeton Oxford: Princeton University Press.

Yendell, Alexander. 2018. Understanding and Explaining Islamophobia in Eastern Europe. EEGA@Connections Special Issue No. 1. Leipzig. https:/www.leibniz-eega.de/wp-content/uploads/2019/03/EEGA_ Special-Issue_1_Islamophobia.pdf. Zugegriffen: 11. Mai 2020.

Yendell, Alexander, und Stefan Huber. 2020. The relevance of the centrality and content of religiosity for explaining Islamophobia in Switzerland. Religions 11(3):129. https://doi.org/10.3390/rel11030129. 